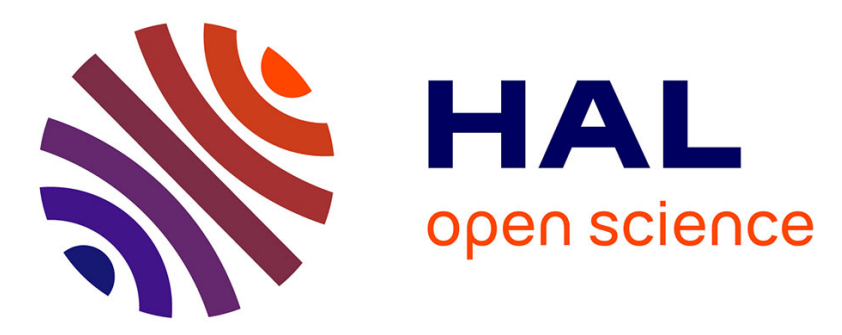

\title{
Atlas-based Transfer of Boundary Conditions for Biomechanical Simulation
}

Rosalie Plantefeve, Igor Peterlik, Hadrien Courtecuisse, Raffaella Trivisonne, Jean-Pierre Radoux, Stéphane Cotin

\section{- To cite this version:}

Rosalie Plantefeve, Igor Peterlik, Hadrien Courtecuisse, Raffaella Trivisonne, Jean-Pierre Radoux, et al.. Atlas-based Transfer of Boundary Conditions for Biomechanical Simulation. MICCAI - 17th International Conference on Medical Image Computing and Computer Assisted Intervention, Sep 2014, Boston, United States. hal-01070339

\section{HAL Id: hal-01070339 \\ https://inria.hal.science/hal-01070339}

Submitted on 1 Oct 2014

HAL is a multi-disciplinary open access archive for the deposit and dissemination of scientific research documents, whether they are published or not. The documents may come from teaching and research institutions in France or abroad, or from public or private research centers.
L'archive ouverte pluridisciplinaire HAL, est destinée au dépôt et à la diffusion de documents scientifiques de niveau recherche, publiés ou non, émanant des établissements d'enseignement et de recherche français ou étrangers, des laboratoires publics ou privés. 


\title{
Atlas-based Transfer of Boundary Conditions for Biomechanical Simulation
}

\author{
R. Plantefève ${ }^{12}$, I. Peterlik ${ }^{34}$, H. Courtecuisse ${ }^{5}$, R. Trivisonne ${ }^{23}$, J-P. Radoux ${ }^{1}$, \\ and S. Cotin ${ }^{23}$ \\ 1 ALTRAN, MEDIC@ (Medical Image Computerized @nalysis), Strasbourg, France \\ 2 INRIA Shacra, Lille, France \\ 3 Institut Hospitalo-Universitaire, Strasbourg, France \\ ${ }^{4}$ Institute of Computer Science, Masaryk University, Brno, Czech Republic \\ 5 CNRS ICube, Strasbourg, France
}

\begin{abstract}
An environment composed of different types of living tissues (such as the abdominal cavity) reveals a high complexity of boundary conditions, which are the attachments (e.g. connective tissues, ligaments) connecting different anatomical structures. Together with the material properties, the boundary conditions have a significant influence on the mechanical response of the organs, however corresponding correct mechanical modeling remains a challenging task, as the connective structures are difficult to identify in certain standard imaging modalities. In this paper, we present a method for automatic modeling of boundary conditions in deformable anatomical structures, which is an important step in patient-specific biomechanical simulations. The method is based on a statistical atlas which gathers data defining the connective structures attached to the organ of interest. In order to transfer the information stored in the atlas to a specific patient, the atlas is registered to the patient data using a physics-based technique and the resulting boundary conditions are defined according to the mean position and variance available in the atlas. The method is evaluated using abdominal scans of ten patients. The results show that the atlas provides a sufficient information about the boundary conditions which can be reliably transferred to a specific patient. The boundary conditions obtained by the atlas-based transfer show a good match both with actual segmented boundary conditions and in terms of mechanical response of deformable organs.
\end{abstract}

\section{Introduction}

The role of numerical simulations in medical training, pre-operative planning and intra-operative guidance has increased considerably in the last decade. While generic anatomical models are employed in training systems, pre-operative planning and intra-operative guidance require patient-specific models. The geometry of such models is usually obtained from pre-operative image data (such as CT), however, two physics-related aspects must be taken into account in order to create a correct biomechanical model: physical properties of organs (such as elasticity parameters) and their boundary conditions which define the actual 
mechanical coupling between different types of living tissues. While in some applications, the exact knowledge of elasticity parameters is not crucial [1] and relative or apparent stiffness is sufficient for correct simulation of tissue behaviour, the correct definition of boundary conditions is always the key aspect in mechanical modeling, since it directly determines the particular solution to the overall physical problem.

The identification of boundary conditions has been studied in the field of structural analysis and computer-aided design. For example, in [2] boundary conditions are identified using a boundary stiffness matrix which is obtained as a solution of characteristic equations formulated for different modes of the object. It is assumed that the object follows a linear elastic law and that the boundary conditions also behave linearly. In [3], the non-linear effects are also taken into account in a method based on non-linear normal modes allowing also object with non-linear response; the method is validated using of a simple beam. In [4], accurate determination of boundary conditions including non-linear effects as friction and slip is presented for a two-dimensional circular plate. While these methods achieve an accurate identification of boundary conditions, they are limited to scenarios where the objects have simple and well-defined boundaries. This is usually not the case for anatomical structures considered in medical simulations where complex boundaries are subject to different types of interactions (bilateral and unilateral constraints with and without friction). The work closest to our objective is described in [5] in the context of a multi-modality registration method for image-guided prostate interventions. In this work, a finite-element patient-specific model is built using preoperative MR data and a set of deformations corresponding to different boundary conditions, and randomly sampled material properties are computed and evaluated statistically using a principal component analysis.

In the area of medical image registration, it is relatively common to use an atlas to register information not available in the target image. This is the case for instance in brain imaging, where a histological atlas containing detailed anatomical information is mapped onto an MRI of the patient to help target small structures during neurosurgery planning [6]. The idea presented there is to use a similar approach to first build an atlas of liver models including the regions where the ligaments are located, and then register the atlas onto the patient to transfer the boundary conditions. In this process, the atlas creation is an important step, as well as the quality of the registration method. The most common type of atlas consists in a mean shape (or image) generated from a database of several shapes (or images). The creation of the atlas involves the segmentation of anatomical structures in each image of the database followed by the registration of each image into a common reference frame. Variations of this process include multiple images of the same patient using different imaging techniques or parameters, or the use of a single image in the database, obtained from a high-resolution imaging process. Statistical atlases rely on a similar idea, but take into account inter-subject anatomical variations. The shape correlations between different anatomical structures are usually described using a conditional 
Gaussian model [7]. A two-step registration process is then required to estimate the probability of having a certain anatomical information in the low resolution or low contrast target image.

The registration process can take many forms, either in the image space or using reconstructed geometrical models. In the following we essentially focus on registration methods applied between surface representations but involving a physics-based approach to estimate a volumetric displacement field. For instance, in [8] a registration of intra-operative MR brain images is proposed where the model is based on linear elasticity and a finite element method. The method is driven by active surface matching which deforms the boundary of brain in one acquired image towards the boundary in the following scan. A multi-organ deformable image registration based on a biomechanical model is presented in [9]. The model driven by surface deformation and displacements of landmarks is used to analyze and predict the motion of abdominal organ during respiration.

In this paper, we propose a combination of a statistical atlas and a physicsbased registration method to estimate boundary conditions in the target space.

\section{Methods}

The main idea of our method is to create a statistical atlas of the structures of interest, incorporating the constraint locations specific to each patient, as well as the mean elastic properties found in the literature [10]. The atlas is then registered onto the patient data using a physics-based approach. In the remainder of this section we consider the liver as the organ of interest, and the ligaments, arteries and veins that "connect" it to the surrounding anatomy as the boundary conditions (see Fig 1).

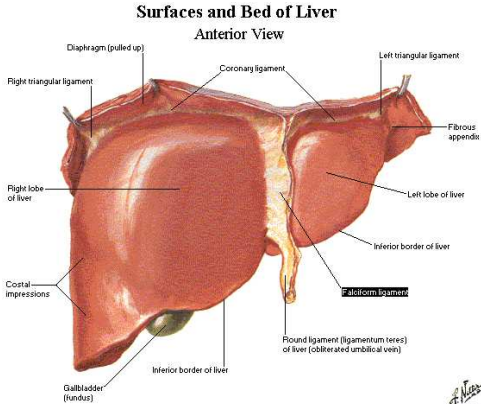

(a) Liver anatomy

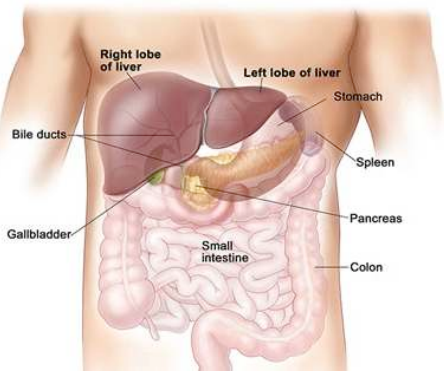

(b) Liver in the abdomen

Fig. 1. Liver is a good example of an organ with mechanical behavior highly influenced by its environment, mainly by bilateral constraints (ligaments and vessels) and unilateral constraints (contacts with the surrounding anatomical structures). 


\subsection{Statistical atlas creation}

The creation process for the statistical atlas is partially based on the work of Wang et al. [7]. It assumes that we have three-dimensional surface models of the different structures of interest (in our case the organ and the regions where the boundary conditions are located). Then a number of points is sampled onto the surface of each mesh. The same number of points and numbering is used for each point cloud. From this set of point clouds, a Generalized Procrustes Analysis (GPA) [11] is performed to determine similarity transformation $S I M_{i}():. S_{i} \rightarrow C$ that aligns best all models of the database into a common reference frame. This transformation is specific for each model $i$, where $S_{i}$ is the space of the segmented model and $C$ is the common reference frame. Then a principal component analysis is performed on each structure of interest (organ and boundary conditions) to compute the principal modes of deformation across the database. We keep only the most significant modes, i.e. the modes responsible for more than $99 \%$ of the deformations. Assuming the Gaussian distribution of the modes, we can determine the mean and standard deviation associated with each structure. The same is done for the elastic modulus of each boundary condition. The technique is summarized in Fig. 2.

\subsection{Atlas to patient registration}

The aim of this step is to find the boundary conditions of a patient-specific organ model. The patient anatomy augmented by the atlas includes a typical stiffness and mean position associated to each boundary condition, and the variance around this mean. Having a 3D model obtained from pre-operative data and the atlas described above, the aim is to compute an elastic transformation which maximizes the shape similarity between the atlas model and the patient specific model. The procedure is done as follows: First the three-dimensional mean shape organ model is registered to the patient data. The registration method is the same as the one used for the atlas construction and is described in the next

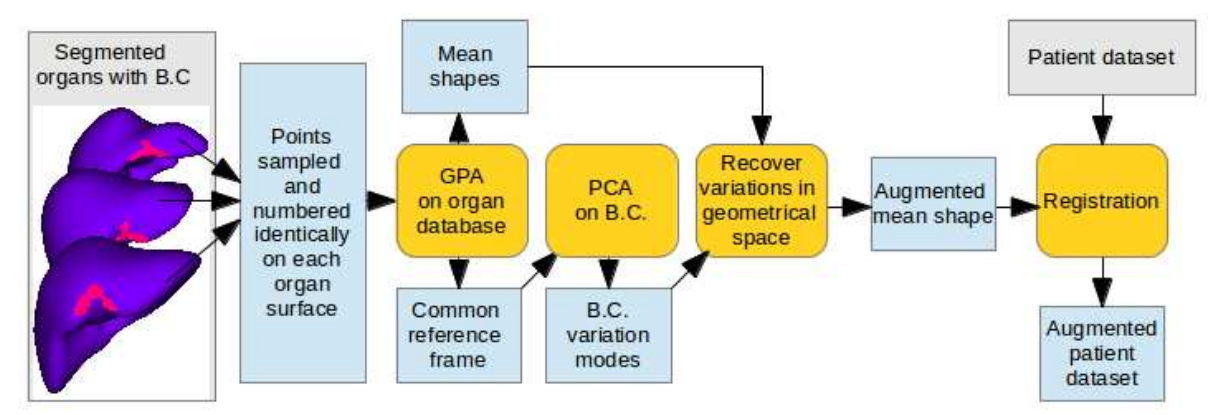

Fig. 2. Main steps of the atlas creation process and its application to the transfer of boundary conditions. 
section. This process also transfers the boundary conditions to the patient data. The standard deviation associated with each boundary condition in the atlas is also transferred to the patient data. However, while the position variability in the atlas is a three-dimensional distribution on the target mesh, it must be projected onto the surface of the mesh (by definition, boundary conditions are only applied to the boundary). The elastic modulus of each boundary condition, available in the atlas, is also associated to this geometrical description.

\subsection{Feature-based Registration}

For both creation of the statistical atlas and transfer of the boundary conditions to a specific patient data, our approach relies on non-rigid registration which computes an elastic transformation which maximizes the shape similarity between the source and target configurations. This critical step has an important influence on the accuracy of the method and must guarantee that corresponding anatomical parts be correctly matched during the registration. We propose a non-rigid registration framework using a feature-based dynamic weighted Iterative Closest Point (ICP). A set of anatomical landmarks is automatically detected on both the source and the target meshes. A finite element simulation is then performed to register the atlas data to the segmented MR image. Anatomical landmarks provide a coarse set of correspondences that improves the robustness of the ICP method, while the biomechanical model is used to regularize the registration process.

Automatic Ridge line Detection: The ridge line is an anatomical structure that is defined as a sharp transition located on the segment $6,5,4 \mathrm{~b}$ and 3 of the liver surface. This landmark can be easily identified in most of human liver segmentations. The automatic detection of the ridge line on the $3 \mathrm{D}$ model of the liver is performed as follows: the edges separating two triangles with sufficiently different normals are selected as seeds. Then, the ridge line is extended from the seed edges; if no extension is found the seeds are removed. Iteratively the ridge line is reconstructed. The ridge line crosses the entire length of the liver and gives a robust landmark for the ICP method.

Biomechanical model: A biomechanical model is associated to the mean shape of the atlas. The deformation of the tissue is modeled with linear tetrahedral finite elements employing the co-rotational formulation handling large displacements correctly [12]. Moreover, although they may be of the same order of magnitude, the patient specific organ do not have necessarily the same volume as the mean shape of the atlas. Therefore the compressibility parameter of the biomechanical model is defined so that variation of volume are allowed during the registration. Definition of constraints: Two ICPs are performed simultaneously (for the anatomical landmarks and for the surface of the organ) from which two sets of constraints are extracted. The constraints are imposed on the system with a penalty force; its energy is given as $E=\sum_{i}^{n_{l}} \frac{1}{2} k_{l} x_{l}^{2}+\sum_{i}^{n_{s}} \frac{1}{2} k_{s} x_{s}^{2}$ where $n$ is the number of constraints, $k$ is a stiffness penalty factor and $x$ is the distance between the source and the target meshes. Subscripts $l$ and $s$ denotes respectively the constraints obtained from the anatomical landmarks and the surface of the 
biomechanical model. Indeed, although the ICP algorithm is fast and easy to implement, the binding process suffers from inaccuracy (in particular for non-rigid registration) which can lead to a energy local minimum. We improve the method by considering that the points belonging to anatomical regions offer higher reliability and must be penalized differently. Therefore, $k_{l}$ is constant while $k_{s}$ is initialized to zero and follows a monotonically increasing function of the form $\frac{k_{s_{\max }(n v)^{2}}}{(n v)^{2}+1}$ over the simulation steps where $n$ is the stem number and $\mathrm{v}$ a speed coefficient. Thus, the constraints applied on the whole surface are negligible at the beginning of the registration and become dominant over time.

Registration method: A non linear problem $\mathbf{K}(\mathbf{u})=\mathbf{f}$ is solved at each simulation step where $u$ is the displacement vector, $\mathbf{K}$ the non-linear stiffness provided by the FE formulation and $\mathbf{f}=\frac{d E(\mathbf{u})}{\mathbf{d u}}$ is the vector of constraint forces. As $k_{s}$ tends to infinity, the solution of the system is equivalent to minimisation of the $\mathcal{L}_{2}$ distance $(x=0)$ between the source and target meshes. Finally, during the simulation the anatomical landmarks provide a coarse registration that improves the robustness of the ICP method, whereas the biomechanical model plays a role of regularization and allows for an accurate solution of local deformations.

\section{Results}

In this section we demonstrate the potential of our method using an atlas and patient specific models of human liver. The atlas is created using ten liver models and their respective anatomical features obtained from segmented abdominal CT scans. The chosen anatomical features are all the liver fixation zones that can be seen on CT images: the falciform ligament, which can be detected thanks to the falciform ligament groove, and the entrance and exit of the hepatic vein.

To compute the statistics of our atlas, we measured the standard deviation $\sigma$ of the ligament and the vein positions in $x, y$ and $z$. For the ligament, we obtain $\sigma_{x}=10.56 \mathrm{~mm}, \sigma_{y}=13.4 \mathrm{~mm}$ and $\sigma_{z}=9.4 \mathrm{~mm}$, whereas for vein, we get $\sigma_{x}=8.4 \mathrm{~mm}, \sigma_{y}=17.0 \mathrm{~mm}$ and $\sigma_{z}=24.3 \mathrm{~mm}$. Given that the size of liver boundingbox is $215 \times 160 \times 110 \mathrm{~mm}^{3}$, the average variability represents less than $9.7 \%$ of the size of the organ showing a strong consistency among the feature positions.

In the registration described in section 2, the Young's modulus of the liver was set to $10 \mathrm{kPa}$. The coefficients $k_{l}$ and $k_{s_{\max }}$ were set to $500 \mathrm{kN} \cdot \mathrm{m}^{-1}$ and $1000 \mathrm{kN} . \mathrm{m}^{-1}$ in order to minimize the registration error while ensuring the numerical stability. The absolute values are not crucial, since the results are not affected as long as the optimal ratio of the organ stiffness to the constraint stiffness is maintained.

In order to evaluate our registration method, we measured the Hausdorff distance between the mean shape of the atlas registered on a liver model. The result obtained for one liver is visualized in Fig. 3(a): the mean error after registration measured by the Hausdorff is $0.5 \mathrm{~mm}$, the maximum error is $6.7 \mathrm{~mm}$. In Fig. 3(b), a visualization of the Hausdorff distance between the estimated boundary conditions and boundary conditions obtained by a manual segmentation of ligaments and veins is presented. In this case, the falciform ligament 


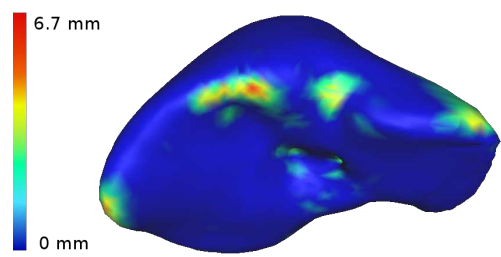

(a)

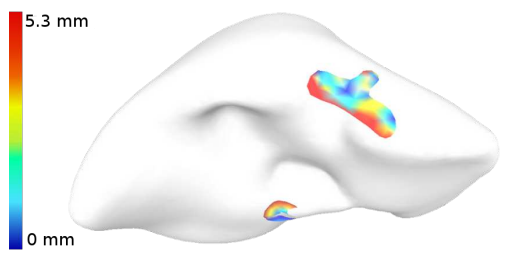

(b)

Fig. 3. (a) Error in registration of the atlas to the patient-specific data. (b) Error between estimated boundary conditions and ground truth obtained by manual segmentation.

and the vein features are recovered with a mean Hausdorff error of $4.2 \mathrm{~mm}$. This value is similar to their position standard deviation in the atlas.

Finally, the method is evaluated by measuring the accuracy of shape prediction in a deformed configuration. A liver segmented in a supine position is used to set up a simulation where gravity is re-oriented in order to simulate a deformation of the organ in a flank position. The Young's modulus were chosen according to the literature [10]: $10 \mathrm{kPa}$ for the liver and $20 \mathrm{MPa}$ for the ligament and the vein. A preliminary parameter sensitivity analysis shows that the results are not affected by small changes of the boundary conditions elasticity. Two simulations are preformed: (i) ground-truth simulation where the liver model is constrained using the boundary conditions obtained from manual segmentation and (ii) atlas-based simulation employing the boundary conditions estimated by our method. In both cases, a very good match between the resulting shapes is

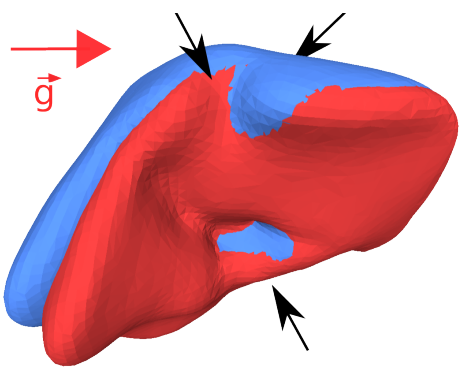

(a)

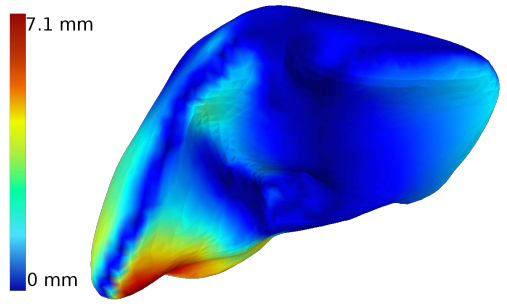

(b)

Fig. 4. Simulation of flank to supine deformation of the liver: (a) shows the displacement between flank and supine position. The black arrows indicate the position of boundary conditions and the red arrow represents gravity; (b) Hausdorff error with boundary conditions constructed using the atlas. 
obtained after the equilibrium is achieved as shown in Fig. 4: the comparison gives the mean error of $1.6 \mathrm{~mm}$ and a maximum error of $7.1 \mathrm{~mm}$.

\section{Conclusion}

In this paper, we proposed a method for automatic modeling of boundary conditions in deformable anatomical structures. The method is based on a statistical atlas which gathers data defining the locations of the connective structures attached to the organ of interest. In order to transfer the information stored in the atlas, a physics-based registration technique is used. The resulting transferred boundary conditions are defined according to the mean position and variance available in the atlas. The method is evaluated using abdominal scans of ten patients. The results show that that the atlas provides a sufficient information about the boundary conditions which can be reliably transferred to a specific patient.

In the future work, we plan to perform further validation on patient data and extend the atlas by considering the unilateral boundary conditions such as contacts with surrounding structures.

\section{References}

1. Miller, K., Lu, J.: On the prospect of patient-specific biomechanics without patientspecific properties of tissues. J Mech Behav Biomed Mater. 27 (2013) 154-166

2. Ahmadian, H., Mottershead, J., Friswell, M.: Boundary condition identification by solving characteristic equations. J. of Sound and Vibration 247(5) (2001) 755-763

3. Ahmadian, H., Zamani, A.: Identification of nonlinear boundary effects using nonlinear normal modes. Mech. Systems and Signal Proc. 23(6) (2009) 2008-2018

4. Suzuki, A., Kamiya, K., Yasuda, K.: Identification technique for nonlinear boundary conditions of a circular plate. J. of Sound and Vibration 289(12) (2006)

5. Hu, Y., Ahmed, H.U., Taylor, Z., Allen, C., Emberton, M., Hawkes, D., Barratt, D.: MR to ultrasound registration for image-guided prostate interventions. Medical image analysis 16(3) (December 2012) 687-703

6. Bardinet, E., et al.: A three-dimensional histological atlas of the human basal ganglia. atlas deformation strategy and evaluation in deep brain stimulation for parkinson disease. J. of Neurosurgery 110(2) (2009)

7. Wang, H., Stout, D.B., Chatziioannou, A.: Estimation of mouse organ locations through registration of a statistical mouse atlas with micro-ct images. IEEE Trans. Med. Imaging 31(1) (2012) 88-102

8. Ferrant, M., Nabavi, A., Macq, B., Jolesz, F.a., Kikinis, R., Warfield, S.K.: Registration of 3-D intraoperative MR images of the brain using a finite-element biomechanical model. IEEE Trans. on Medical Imaging 20(12) (2001) 1384-1397

9. Brock, K., Sharpe, M., Dawson, L., Kim, S., Jaffray, D.: Accuracy of finite element model-based multi-organ deformable image registration. Med Physics 32(6) (2005)

10. Umale, S.: Characterization and modeling of abdominal organs. PhD thesis, University of Strasbourg (2012)

11. Gower, J.C.: Generalised Procrustes Analysis. Psychometrika 40 (1975) 33-51

12. Felippa, C., Haugen, B.: A unified formulation of small-strain corotational finite elements: I. theory. Comput Meth Appl Mech Eng 194(21) (2005) 